\title{
A Conceptual Framework to Predict Mental Health Patients' Zoning Classification
}

\author{
Sanjib Raj PANDEY ${ }^{\mathrm{a}}$, Alan $\mathrm{SMITH}^{\mathrm{b}}$, Edmund Nigel GALL ${ }^{\mathrm{a}}$, Ajay BHATNAGAR ${ }^{\mathrm{c}}$ \\ and Thierry CHAUSSALET ${ }^{\mathrm{a}, 1}$

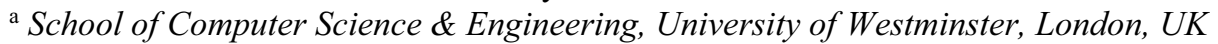 \\ ${ }^{\mathrm{b}}$ Business Intelligence Systems Team, Oxleas NHS Foundation Trust, London, UK \\ ${ }^{\mathrm{c}}$ Green Parks House (Inpatient Services), Oxleas NHS Foundation Trust, London, UK
}

\begin{abstract}
Zoning classification is a rating mechanism, which uses a three-tier color coding to indicate perceived risk from the patients' conditions. It is a widely adopted manual system used across mental health settings, however it is time consuming and costly. We propose to automate classification, by adopting a hybrid approach, which combines Temporal Abstraction to capture the temporal relationship between symptoms and patients' behaviors, Natural Language Processing to quantify statistical information from patient notes, and Supervised Machine Learning Models to make a final prediction of zoning classification for mental health patients.
\end{abstract}

Keywords. mental health, zoning, temporal logic, natural language processing, machine learning

\section{Introduction}

Mental health (MH) is the biggest cause of disability in the UK: its estimated economic and social cost of $£ 105.2$ billion annually contributes up to $22.8 \%$ of the total expenditure [1]. Identifying, assessing, and mitigating mental health risk factors holds the potential to reduce the cost burden by correctly proportioning interventions according to the assessed risk. UK mental health trusts (MHTs) use a mostly evidenced-based manual process for zoning classification, a clinical assessment of risk factors to assign patients to zones using established zoning criteria [2]. This provides essential and pragmatic support to the Health Care Professionals (HCPs) who are required to regularly assess, implement, and evaluate the treatment plans of the MH patients in their care. In this study we introduce an automated framework to facilitate the design and development of an evidence-based decision support tool to predict and inform $\mathrm{MH}$ zoning classification.

Patients are diagnosed as suffering from one or more $\mathrm{MH}$ conditions as described by the International Classification of Diseases Version 10 (ICD-10) [3]. Within each ICD-10 category a patient may experience a series of symptoms and behaviors, each with defined start and end time points (episodes), of varying intensities that describe the severity of the impact the episode has on the patient's wellbeing. By combining episode profile of ICD-10 descriptors, intensity, and environmental triggers, a time-based overall descriptor of patient acuity can be derived. The zoning process aims to classify the patient

\footnotetext{
${ }^{1}$ Corresponding Author, Thierry Chaussalet, School of Computer Science \& Engineering, University of Westminster, 115 New Cavendish St, London, W1W 6UW, UK; E-mail: chausst@westminster.ac.uk.
} 
into one of three risk levels (Red: High; Amber: Medium; and Green: Low), based on a set of guidelines that map changing patient acuity to changing risks, during regular meetings, where discussions of patient condition relative to risk ensure that appropriate care management plans are in place. However, issues were identified regarding the current zoning approach. First, there is no real time reflection of caseload and patients' risk profiles. Second, manual assessments are resource intensive. Third, subjective assessment can result in inconsistent ratings. Fourth, manual practices impede on optimal sharing of this key information with all staff.

There are various approaches to extract clinical data for automated predictions from Electronic Health Records (EHRs). A hybrid approach comprising supervised Machine Learning (ML), rule-based annotation, and unsupervised natural language processing (NLP) for identifying disorder mentions achieved a 0.776 F-score [4]. Zhang et al. [5] proposed a system incorporating temporal modelling, and ML approaches for an early anxiety/depression detection framework. Neural networks and NLP were applied to predict the presence/absence of MH conditions using clinical notes [6]. NLP was used to capture severe mental illness symptoms from clinical text within discharge summaries [7]. Clinical data extraction divides into two categories: (a) ML and rule-based approaches, and (b) information extraction applications are both discussed in [8].

This research will investigate techniques required to develop a tool and assess the feasibility of its use within an NHS MHT, as a replacement/augmentation of the current system. The tool should have flexibility to allow Trusts to tailor it to their requirements.

\section{Methodology}

We propose a hybrid approach to build an automated decision support tool that will classify patient zoning stages, comprising a Temporal Model, an NLP Model and Supervised ML Model, as shown in Figure 1. Each model's specific responsibilities and functionalities are discussed below.

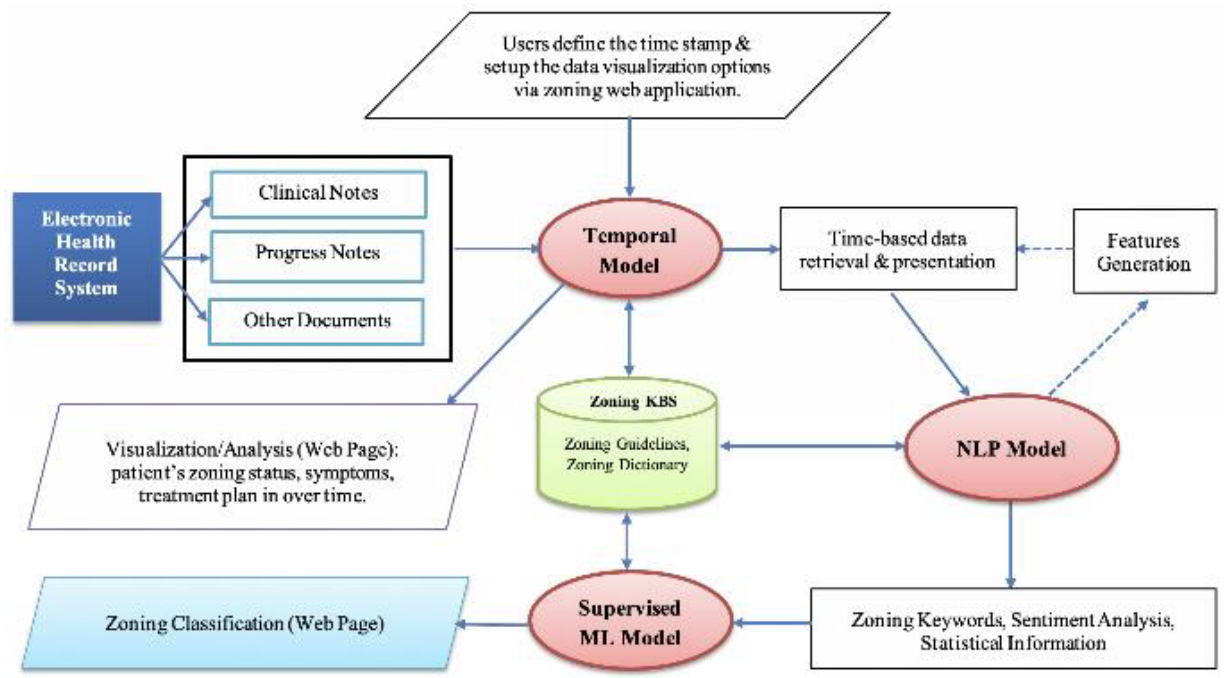

Figure 1. Hybrid approach for automated decision support tool to predict and inform zoning classification. 
Both absolute time (the date and time of an event) and relative time (the intervals between events), respectively known as Point-based and Interval-based temporal approaches, are key elements of clinical data management. In this framework we propose using point-based temporal primitives, which are good for computation and more appropriate for the clinical reasoning process, to derive the Temporal Model, a temporal abstraction and representation of zoning classification. This shall feed the NLP Model, as shown in Figure 2. The NLP Model performs the following tasks: keywords extraction based on the Zoning Knowledge-based System (KBS); sentiment analysis (e.g., with the contextual window); and calculates statistical information about the zoning keywords.

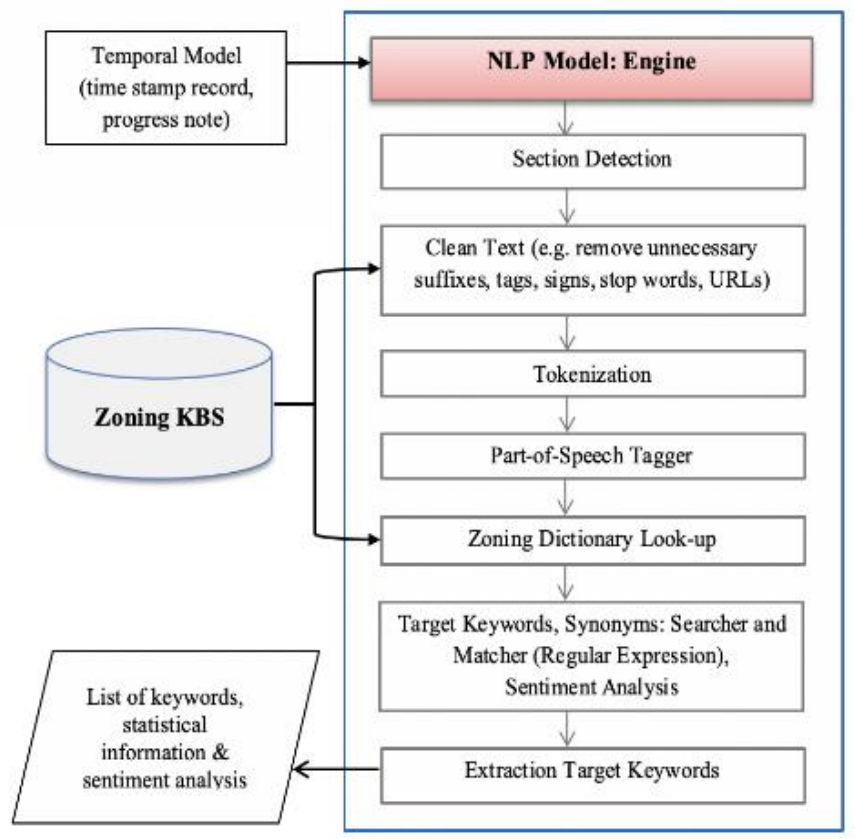

Figure 2. The NLP Model Process.

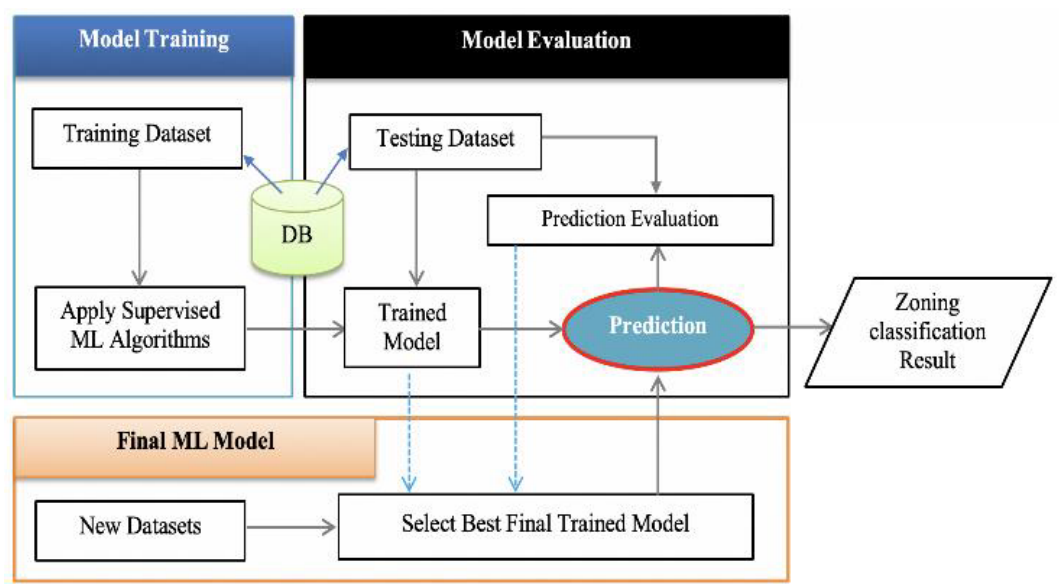

Figure 3. The Supervised ML Model Process. 
The Supervised ML Model, shown in Figure 3, reads the target keywords, sentiment analysis and statistical information from the NLP Model, generates required ML and prediction datasets, produces the final tuned $\mathrm{ML}$ model, and predicts zoning classifications. We will use several supervised ML algorithms, as have already been applied in medical information applications, e.g. logistic regression, decision tree, support vector machine, neural network, and random forest [9]. The output of each algorithm will be evaluated; one algorithm will be selected for the final development based on the evaluated results.

\section{Conclusion and Future Work}

In this paper we introduced a new conceptual framework using a hybrid approach, combining Temporal, Natural Language Processing, and Supervised Machine Learning Models for patient zoning classification in mental health. Ultimately, we will develop a real-time artificial intelligence-based zoning classification tool for treatment of mental health patients, in collaboration with domain users (e.g., doctors, nurses, other HCPs and ex-patients) to ensure the project meets their needs whilst applying our proposed hybrid approach.

\section{References}

[1] HMG/DH. No health without mental health: a cross-government mental health outcomes strategy for people of all ages [Internet]. London: Department of Health; 2011 Feb [cited 2021 Feb 11]. 103 p. Available from: https:/www.gov.uk/government/publications/no-health-without-mental-health-a-crossgovernment-outcomes-strategy.

[2] Croucher S, Williamson GR. Risk assessment in mental health: Introducing a traffic light system in a community mental health team. The Open Nursing Journal. 2013;7:82-8. doi:10.2174/1874434620130529004.

[3] World Health Organization, editor. The ICD-10 classification of mental and behavioural disorders: clinical descriptions and diagnostic guidelines [Internet]. Geneva: World Health Organization; 1992 [cited 2021 Jan 12]. 362 p. Available from: https://apps.who.int/iris/handle/10665/37958

[4] Wang C, Akella R. A Hybrid Approach to Extracting Disorder Mentions from Clinical Notes. AMIA Jt Summits Transl Sci Proc. 2015 Mar 25 [cited 2021 Mar 4];2015:183-7. Cited in PubMed; PMID 26306265.

[5] Zhang J, Xiong H, Huang Y, Wu H, Leach K, Barnes LE. M-SEQ: Early detection of anxiety and depression via temporal orders of diagnoses in electronic health data. In: 2015 IEEE International Conference on Big Data (Big Data). 2015;2569-77. doi:10.1109/BigData.2015.7364054.

[6] Tran T, Kavuluru R. Predicting mental conditions based on "history of present illness" in psychiatric notes with deep neural networks. Journal of Biomedical Informatics. 2017 Nov 1;75:S138-48. doi:10.1016/j.jbi.2017.06.010.

[7] Jackson RG, Patel R, Jayatilleke N, Kolliakou A, Ball M, Gorrell G, Roberts A, Dobson RJ, Stewart R. Natural language processing to extract symptoms of severe mental illness from clinical text: the Clinical Record Interactive Search Comprehensive Data Extraction (CRIS-CODE) project. BMJ open. 2017 Jan 1;7(1):e012012.

[8] Wang Y, Wang L, Rastegar-Mojarad M, Moon S, Shen F, Afzal N, Liu S, Zeng Y, Mehrabi S, Sohn S, Liu H. Clinical information extraction applications: a literature review. Journal of biomedical informatics. 2018 Jan 1;77:34-49.

[9] Pandey SR, Ma J, Lai CH, Regmi PR. A supervised machine learning approach to generate the auto rule for clinical decision support system. Trends in Medicine. 2020 May 22;20(3):1-9. doi:10.15761/TiM.1000232. 